\title{
Determination of the Wilson ChPT low energy constant $c_{2}$
}

\author{
Fabio Bernardoni ${ }^{* \dagger}$ \\ NIC, DESY, Zeuthen \\ E-mail: fabiobeifh.de

\section{John Bulava} \\ NIC, DESY, Zeuthen \\ E-mail: john.bulava@desy.de

\section{Rainer Sommer} \\ NIC, DESY, Zeuthen \\ E-mail: sommereifh.de
}

Following a suggestion by Aoki and Bär, $c_{2}$ can be extracted by analyzing volume effects in 2 pion states. To this end we consider renormalized ratios of four point to two point correlation functions. We present the results from various CLS lattices, with pion masses ranging from 280 to $450 \mathrm{MeV}$ and lattice spacings of $0.07 \mathrm{fm}$ and $0.08 \mathrm{fm}$. This low energy constant is useful to understand discretization effects in chiral extrapolations with Wilson fermions, especially for quantities which vanish in the chiral limit, like the pion mass. Since our procedure is computationally cheap and straightforward, it is recommended as a routine study for any Wilson-type simulation, as a check of discretization effects.

DESY 11-209

SFB/CPP-11-67

The XXIX International Symposium on Lattice Field Theory - Lattice 2011

July 10-16, 2011

Squaw Valley, Lake Tahoe, California

\footnotetext{
* Speaker.

${ }^{\dagger}$ We thank O. Bär, M. Lüscher, S. Necco and F. Virotta for useful discussions.
} 


\section{Introduction}

For a few years we have had algorithms that allow simulation at small quark masses $m$ in large volumes, such that $M_{\pi} L \gtrsim 4$. As a consequence of this progress, and since volume effects are exponentially suppressed in $M_{\pi} L$, they are presently kept at the level of $1 \%$. On the other hand, discretization effects obey power law scaling, which in the case of $O(a)$ improved Wilson fermions amount to $O\left(a^{2}\right)$ effects. Most importantly, for all kinds of Wilson fermions, the dominant discretization effects are related to the breaking of chiral symmetry. As a consequence, they become more relevant as we try to reach lower quark masses at fixed $a$. It is then clear that the understanding and removal of discretization effects is essential.

Recent studies [1] however have shown a severe critical slowing down which makes it unrealistic to simulate lattices with $a \lesssim 0.05 \mathrm{fm}$. While the critical exponent can be reduced from $\sim 5$ to 2 by using open boundary conditions [2], to reduce the lattice spacing remains challenging and an understanding of the discretization effects is necessary to establish trustworthy results.

One framework to study the discretization effects is provided by Wilson ChPT (WChPT) [3], the chiral effective theory of Wilson LQCD. In this effective theory the cutoff effects are parametrized at a given order in the chiral expansion by a finite number of Low Energy Constants (LECs), among which we find $c_{2}$. In terms of the physical and unphysical LECs, Wilson ChPT predicts the scaling of pion observables with respect to $m$ and $a$. In this context, for one class of observables, $c_{2}$ plays an important role as it appears in the LO lagrangian.

In this talk we present our results and strategy to put at least an upper limit on $\left|c_{2}\right|$ and eventually establish its sign. We also discuss the relevance that the values found would have on the $m$-dependence of some physical observables.

\section{Wilson ChPT}

The first step to build WChPT is to collect all the operators in the continuum Symanzik effective theory that are compatible with the symmetries of Wilson LQCD up to a certain dimension (i.e. power in $a$ ). These then have to be matched to the chiral effective theory; they must be rewritten in terms of pion fields. Once a power counting for $a$ relative to $m$ and $p$ has been established, the spurion technique which was already used to introduce chiral symmetry breaking effects due to $m$ can be extended to treat cutoff effects.

Our aim is to demonstrate that cutoff effects are not strong enough to distort the physical chiral scaling of the observables in which we are interested. As a consequence, we measure on our coarser lattices with $a=0.075 \mathrm{fm}$ and $a=0.065 \mathrm{fm}$ and assume a conservative power counting for $a$ (the so called LCE regime) which assumes:

$$
m \sim a^{2} \Lambda_{Q C D}^{3} \quad a \simeq 0.07 \mathrm{fm} \Rightarrow a^{2} \Lambda_{Q C D}^{3} \sim 10 \mathrm{MeV} .
$$

This counting is expected to be the proper one for quark masses around $10 \mathrm{MeV}$. However large or small coefficients in the expansion could shift this limit up or down so a practical test is necessary. We also assume (which is true in our case) that $O(a)$ improvement has been carried out nonperturbatively. 
With the power counting in eq. (2.1), the LO chiral Lagrangian is:

$$
\mathscr{L}_{\chi}=\frac{F^{2}}{4} \operatorname{Tr}\left[\partial_{\mu} U \partial_{\mu} U^{\dagger}\right]-\frac{\Sigma m}{2} \operatorname{Tr}\left[U+U^{\dagger}\right]+\frac{c_{2} F^{2} a^{2}}{16} \operatorname{Tr}\left[U+U^{\dagger}\right]^{2}
$$

where $c_{2}$ is a new unphysical LEC. One can show that the pion mass is corrected at LO:

$$
M_{\pi}^{2}=M_{0}^{2}+O\left(M_{0}^{4}\right) \quad M_{0}^{2}=\frac{2 \Sigma m}{F^{2}}-2 c_{2} a^{2}
$$

and that a new phase pattern emerges [4], whose properties are controlled by the sign of $c_{2}$ [3]. For Wilson fermions, if $c_{2}>0$ it is possible to reach a phase where flavor and parity are spontaneously broken by decreasing the quark mass at fixed $a$. Since the transition is of second order, the pions are massless on the boundary. Conversely, if $c_{2}$ is negative, the transition is first order, flavor and parity remain unbroken, and there are no massless pions at finite $a$.

We now possess a tool to predict the scaling on $m$ and $a$ (up to a given order) in terms of the physical LECs of ChPT plus some new ones (including $c_{2}$ ). It should be noted that $m$ appearing in the Lagrangian (Eq. (2.2)) is just a bare parameter, which has no immediate relation to a welldefined quark (or pion) mass of the lattice theory. One example of such a quark mass is the PCAC mass, $m_{P C A C}$. Before the WChPT formulas can be used, it is necessary to find a relation between $m$ and $m_{P C A C}$ (or $M_{\pi}$ ) that allows to rewrite the observable of interest $\mathscr{O}$ :

$$
\tilde{\mathscr{O}}\left(m_{P C A C}\right)=\mathscr{O}\left(m_{\left.\left(m_{P C A C}\right)\right)} \text { or } \quad \tilde{\mathscr{O}}\left(M_{\pi}\right)=\mathscr{O}\left(m\left(M_{\pi}\right) .\right.\right.
$$

For example, one can obtain the pion mass as a function of $M_{0}$ (or $m$ through Eq. (2.3)) [5] $]^{1}$ :

$$
M_{\pi}^{2}=M_{0}^{2}\left[1+\frac{M_{0}^{2}+10 c_{2} a^{2}}{64 \pi^{2} F^{2}} \ln \left(\frac{M_{0}^{2}}{\tilde{\mu}_{1}^{2}}\right)+O\left(a^{2}, m\right)\right]+O\left(a^{4}\right),
$$

but this formula cannot be used for extrapolations until a relation between $M_{0}$ and a measurable mass is found. Unfortunately we know just the LO relation between $m$ and $m_{P C A C}$ [6]:

$$
m_{P C A C}=\left(m-\frac{c_{2} a^{2} F^{2}}{\Sigma}\right)\left(1+O\left(a^{2}, m\right)\right) .
$$

After the adequate substitutions are performed, one finds that observables can be separated in two classes, according to how much they are affected by discretization effects.

- Observables that are nonzero in the chiral limit, such as the pion decay constant. Cutoff effects appear here just as analytic contributions and at NLO, both in terms of $M_{\pi}$ and $m_{P C A C}$ :

$$
F_{\pi}^{2}=F^{2}\left[1-\frac{M_{\pi}^{2}}{8 \pi^{2} F^{2}} \ln \frac{M_{\pi}^{2}}{\tilde{\mu}_{4}^{2}}+O\left(a^{2}, m\right)\right]
$$

- Observables that vanish in the chiral limit, such as the pion mass or scattering lengths. Cutoff effects appear at LO, they change the coefficient of the chiral logarithms in such a way that the expansion breaks down at low $M_{\pi}$. Here we show the $S$-wave scattering length for $I=2$, $a_{0}^{2}$, as an example, that will also be useful in the following:

$$
M_{\pi} a_{0}^{2}=-\frac{M_{\pi}^{2}}{16 \pi F_{\pi}^{2}}\left[1+\frac{3 M_{\pi}^{2}+12 c_{2} a^{2}}{32 \pi^{2} F_{\pi}^{2}} \ln \frac{M_{\pi}^{2}}{\tilde{\mu}_{2}^{2}}+O\left(a^{2}, m\right)\right]-\frac{2 c_{2} a^{2}}{16 \pi F_{\pi}^{2}}\left[1+\frac{11 c_{2} a^{2}-2 M_{\pi}^{2}}{16 \pi^{2} F_{\pi}^{2}} \ln \frac{M_{\pi}^{2}}{\tilde{\mu}_{3}^{2}}+O\left(a^{2}, m\right)\right] .
$$

\footnotetext{
${ }^{1}$ Here and in the following we do not write the analytic terms (polynomial in $M_{\pi}^{2}$ ) explicitly for simplicity, but they have also been calculated.
} 


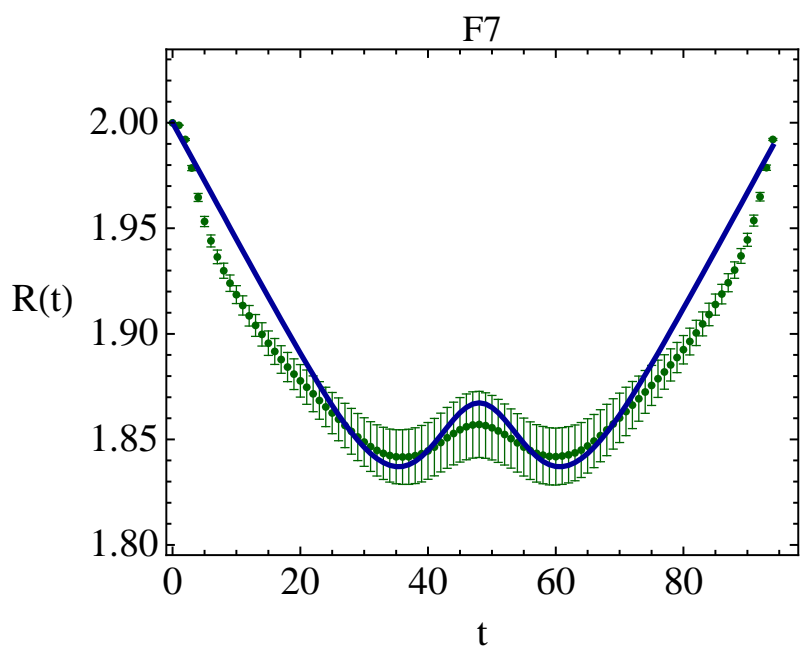

Figure 1: R(t) for the F7 ensemble. The blue curve represents the WChPT prediction.

\section{Determination of $c_{2}$}

From Eqs. (2.6) and (2.3) it is apparent that in the relation $M_{\pi}^{2}\left(m_{P C A C}\right), c_{2}$ appears only at NLO. Conversely $c_{2}$ enters at LO in the relation between the S-wave scattering length and $M_{\pi}$, see Eq. (2.8). The energy eigenvalues of interacting particles are affected in finite volume by a term proportional to the scattering length according to the Lüscher formula:

$$
E_{2 \pi}(\mathbf{p}=0)=2 M_{\pi}+\Delta E \quad \Delta E \equiv-\frac{4 \pi a_{0}^{I}}{M_{\pi} L^{3}}+O\left(L^{-4}\right)
$$

which opens up the possibility to measure $c_{2}$ through volume effects.

We consider the ratio of correlators:

$$
R(t) \equiv \frac{\left\langle\pi^{+}(t) \pi^{+}(t) \pi^{-}(0) \pi^{-}(0)\right\rangle}{\left\langle\pi^{+}(t) \pi^{-}(0)\right\rangle^{2}} \underset{T \rightarrow \infty}{\stackrel{t \gg 1 / M}{\longrightarrow}} e^{-\Delta E t}
$$

where $\pi^{ \pm}(t) \equiv \sum_{\mathbf{x}} \pi^{ \pm}(t, \mathbf{x})$. Apart from the QCD coupling and quark masses, $R(t)$ needs no renormalization and at LO in ChPT it depends only on $F_{\pi}, M_{\pi}$ and $c_{2}$. The result for $R(t)$ on one of our lattices is shown in Fig. 1.

We have evaluated $R(t)$ on the coarser CLS lattices listed in table 1 and in WChPT, in the LCE regime and finite volume. We evaluate all-to-all quark propagators stochastically, using 4 fully time diluted noise sources per configuration. Using $F_{\pi}$ and $M_{\pi}$ determined from the two point correlation functions, we invert the ChPT formula to obtain an effective $c_{2}(t)$. As shown in Fig. 2 we then average over a time interval $\left[t_{\min }, t_{\max }\right]$ such that $t_{\min }>k / M_{\pi},\left(T-t_{\max }\right)>k / M_{\pi}$ for fixed $k=\mathrm{O}(1)$. In practice we have checked that the result does not change significantly for $k \in[2,3]$ and in the following we report the results obtained for $k=2.5$. This definition converges to the LEC $c_{2}$ in the chiral limit. We treat errors coming from autocorrelations in a conservative way, using the techniques described in [1]. To estimate the systematic effect coming from truncating the chiral expansion at LO we compare the results using in the chiral formulas both the observed pion decay constant $F_{\pi}$ and its value in the chiral limit $F_{0}$, see Fig. 2. 


\begin{tabular}{|c|c|c|c|c|}
\hline label & $a(f m)$ & $M_{\pi}(\mathrm{MeV})$ & $M_{\pi} \mathrm{L}$ & $N_{\text {cfg }}$ \\
\hline & & & & \\
$\mathrm{A}_{4}$ & 0.075 & 380 & 4.1 & 100 \\
$\mathrm{~A}_{5}$ & 0.075 & 330 & 4.7 & 325 \\
$\mathrm{E}_{5}$ & 0.065 & 440 & 4.7 & 999 \\
$\mathrm{~F}_{6}$ & 0.065 & 310 & 5 & 300 \\
$\mathrm{~F}_{7}$ & 0.065 & 270 & 4.2 & 600 \\
\hline
\end{tabular}

Table 1: Lattices on which $R(t)$ was computed. Lattices used for physical measurements have lattice spacings as small as $a=0.05 \mathrm{fm}$. The lattice spacing was set from $f_{K}=155 \mathrm{MeV}$, see contribution by $\mathrm{M}$. Marinkovic at this conference.
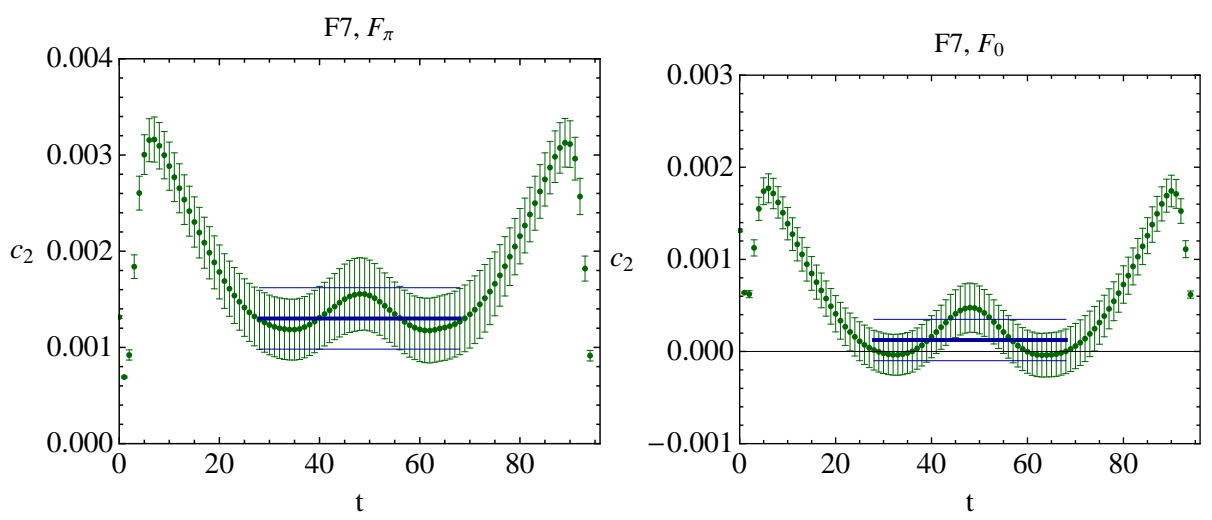

Figure 2: The effective $c_{2}(t)$ with the observed decay constant $F_{\pi}$ (left) and its value in the chiral limit (right).

The results that we obtained are summarized in Fig. 3. Both determinations (using $F_{\pi}$ and using $F_{0}$ ) seem to converge to a positive value, and all our points lie far from the dangerous region (the blue line) where the physical (due to $M_{\pi} \neq 0$ ) and unphysical (due to $a \neq 0$ ) contributions to the S-wave $I=2$ scattering length are equal. It is interesting to mention that the ETMC collaboration found a negative value [7], which is not in contradiction with our result, since their action was different.

\section{Conclusions and Outlook}

Our aim is to find whether or not we expect large cutoff effects in our simulations. Considering that we have performed tests on our coarser lattices and that we have assumed a pessimistic power counting, Fig. 3 is a good indication that cutoff effects are small in our observables and the physical scaling with $M_{\pi}$ (or $m_{P C A C}$ ) is not significantly distorted for the masses that we are considering. Our procedure is cheap and simple, and will be repeated in the future for all ensembles.

We have significant errors, coming mainly from the chiral truncation, that make it difficult to establish a conclusive value for $c_{2}$, although our data at $a=0.065 \mathrm{fm}$ point to a rather small and 

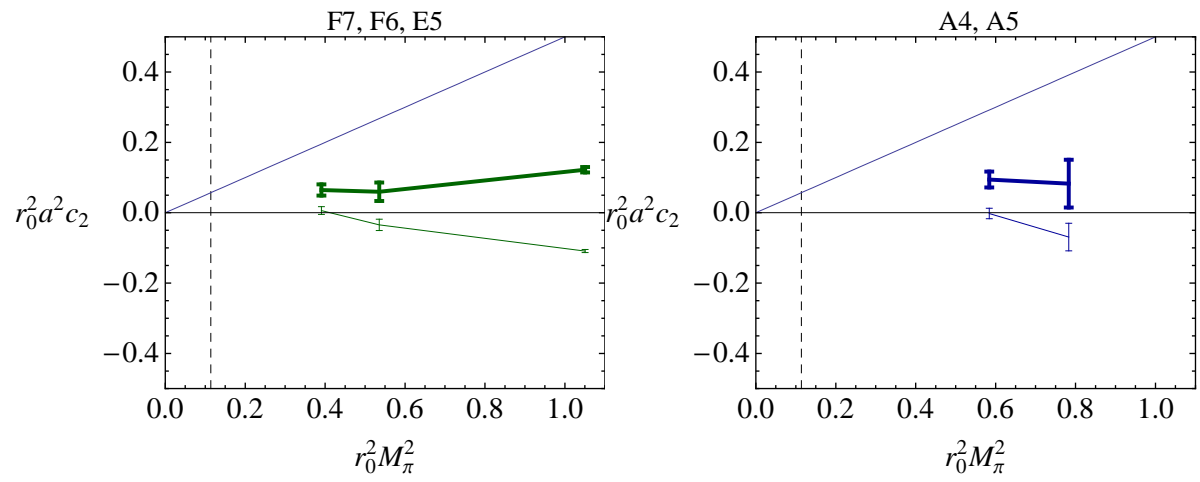

Figure 3: Results for $c_{2}$ on lattices with $a=0.065 \mathrm{fm}$ (left) and $a=0.075 \mathrm{fm}$ (right). Thick lines corresponds to $F_{\pi}$ while thin lines corresponds to $F_{0}$. The dashed line indicates the physical point, the blue line corresponds to $2 c_{2} a^{2}=M_{\pi}^{2}$, which is where the physical contribution to the scattering length is of the same magnitude of the one coming from cutoff effects.

possibly positive value.

In the near future we will apply the same procedure to lattices with smaller masses than the ones so far considered. These additional points may help to establish the sign of $c_{2}$. If indeed $c_{2} \geq 0$ with our action, this would suggest adding a clover term to the action used by the ETM collaboration also yields $c_{2} \geq 0$ [8]. This is important, because in that context $M_{\pi^{ \pm}}-M_{\pi^{0}} \propto c_{2}$ and a negative $c_{2}$ means large volume effects through $M_{\pi^{0}}<M_{\pi^{ \pm}}$.

\section{References}

[1] ALPHA Collaboration, S. Schaefer, R. Sommer and F. Virotta, Nucl.Phys. B845 (2011) 93, 1009.5228.

[2] M. Luscher and S. Schaefer, JHEP 1107 (2011) 036, 1105.4749, * Temporary entry *.

[3] S.R. Sharpe and J. Singleton, Robert L., Phys.Rev. D58 (1998) 074501, hep-lat/9804028.

[4] S. Aoki, Phys.Rev. D30 (1984) 2653.

[5] S. Aoki, O. Bar and B. Biedermann, Phys.Rev. D78 (2008) 114501, 0806.4863.

[6] O. Bar, S. Necco and S. Schaefer, (2008), 0812.2403,

[7] ETM Collaboration, C. Michael and C. Urbach, PoS LAT2007 (2007) 122, 0709.4564.

[8] ETM collaboration, P. Boucaud et al., Comput.Phys.Commun. 179 (2008) 695, 0803.0224. 